\title{
Fluctuations and Landau-Devonshire expansion for Barium Titanate
}

\author{
A. I. Sokolov ${ }^{1}$, A. K. Tagantsev ${ }^{2}$ \\ ${ }^{1}$ Saint Petersburg Electrotechnical University, St. Petersburg 197376, Russia; \\ e-mail:ais@sokol.usr.etu.spb.ru \\ ${ }^{2}$ Laboratoire de Céramique, EPFL, CH-1015 Lausanne, Switzerland; \\ e-mail: Alexander.Tagantsev@epfl.ch
}

(February 20, 2002)

\begin{abstract}
The experimentally observed temperature dependence of the quartic coefficients in the Landau-Devonshire expansion for $\mathrm{BaTiO}_{3}$ is naturally accounted for within a proper fluctuation model. It is explained, in particular, why one of the quartic coefficients varies with temperature above $T_{c}$ while the second is constant. The tetragonal phase in $\mathrm{BaTiO}_{3}$ is argued to exist essentially due to the thermal fluctuations, while the true Landau-Devonshire expansion with temperature-independent coefficients favours the rhombohedral ferroelectric phase. Certain conclusions concerning the temperature dependence of the sextic Landau-Devonshire coefficients are also made.
\end{abstract}

PACS numbers: 77.80.Bh, 77.84.Bw

Typeset using REVTEX 
The phase diagram of the barium titanate contains three lines of phase transitions and its structure is known to be properly reproduced by the phenomenological Landau-Devonshire theory [1]. It is accepted within the phenomenological approach that all the coefficients in the Landau free energy expansion should be either constant or weak functions of temperature, pressure, etc., apart from the quadratic term that changes sign crossing the second-order transition line or the low-temperature spinodal. For the barium titanate, however, the matching with the theory of the experimentally observed temperature dependences of the nonlinear dielectric susceptibility and spontaneous polarization forces one to allow for the strong temperature dependence of some higher-order coefficients [2 [4]. Being in conflict with the spirit of the Landau theory itself, rapid temperature variation of the quartic and sextic coefficients is also quite unexpected from the microscopic point of view. Indeed, the barium titanate is a displacive ferroelectric with weak anharmonicity that can only results in rather slow temperature dependence of the macroscopic parameters [5]. Moreover, there is an extra point showing how unsatisfactory the real situation is: because of the fast temperature variation of the certain coefficient, the six-order Landau-Devonshire expansion for $\mathrm{BaTiO}_{3}$ with updraded coefficients [6.77 turns out to loose its global stability at $T=443 \mathrm{~K}$, i. e. at the temperature that exceeds the cubic-tetragonal transition point $T_{c}$ by only $50 \mathrm{~K}$.

In this Letter, we will show that the temperature dependence of the quartic LandauDevonshire coefficients, experimentally observed in a paraelectric phase of $\mathrm{BaTiO}_{3}$, can be attributed to the thermal fluctuations of polarization and naturally accounted for within a proper fluctuation model. It will be explained, in particular, why in experiments one of the quartic coefficients demonstrates well-pronounced variation with temperature while the second is temperature independent. The conjecture will be put forward concerning the structure of the true Landau-Devonshire form for $\mathrm{BaTiO}_{3}$ with the constant (temperature independent) coefficients, and the first-order fluctuation corrections to the sextic LandauDevonshire coefficients for $T>T_{c}$ will be found.

Barium titanate is a displacive ferroelectric undergoing the first-order ferroelectric phase transitions, with the six-order anharmonicity and electrostriction playing an essential role in 
forming its phase diagram. The electrostriction is known to convert the second-order transition, appropriate to the clamped crystal, into the first-order one. Strong dipole-dipole interaction affects the vibrational spectrum of $\mathrm{BaTiO}_{3}$, resulting in a big gap between transversal (soft) and longitudinal polarization modes. These main features are properly described by the effective Hamiltonian that is the natural generalization of the Landau-Devonshire freeenergy expansion:

$$
\begin{gathered}
H=\frac{1}{2} \sum_{q}\left[\left(\frac{T-T_{0}}{C \epsilon_{0}}+s^{2} q^{2}\right) \delta_{\alpha \beta}+\Delta^{2} n_{\alpha} n_{\beta}\right] \phi_{\alpha q} \phi_{\beta q}^{*}+\frac{1}{2} \int d^{3} x\left[c_{11}\left(u_{11}^{2}+u_{22}^{2}+u_{33}^{2}\right)\right. \\
\left.+2 c_{12}\left(u_{22} u_{33}+u_{33} u_{11}+u_{11} u_{22}\right)+c_{44}\left(u_{12}^{2}+u_{23}^{2}+u_{31}^{2}\right)\right]+H_{i n t}+H_{s t r}, \\
H_{\text {int }}=\int d^{3} x\left[\beta_{1}\left(\phi_{1}^{4}+\phi_{2}^{4}+\phi_{3}^{4}\right)+2 \beta_{2}\left(\phi_{1}^{2} \phi_{2}^{2}+\phi_{2}^{2} \phi_{3}^{2}+\phi_{3}^{2} \phi_{1}^{2}\right)+\gamma_{1}\left(\phi_{1}^{6}+\phi_{2}^{6}+\phi_{3}^{6}\right)\right. \\
\left.+3 \gamma_{2}\left(\phi_{1}^{4} \phi_{2}^{2}+\phi_{1}^{2} \phi_{2}^{4}+\phi_{2}^{4} \phi_{3}^{2}+\phi_{2}^{2} \phi_{3}^{4}+\phi_{3}^{4} \phi_{1}^{2}+\phi_{3}^{2} \phi_{1}^{4}\right)+6 \gamma_{3} \phi_{1}^{2} \phi_{2}^{2} \phi_{3}^{2}\right], \\
H_{s t r}=\int d^{3} x\left\{q_{11}\left(u_{11} \phi_{1}^{2}+u_{22} \phi_{2}^{2}+u_{33} \phi_{3}^{2}\right)+q_{12}\left[u_{11}\left(\phi_{2}^{2}+\phi_{3}^{2}\right)+u_{22}\left(\phi_{3}^{2}+\phi_{1}^{2}\right)\right.\right. \\
\left.\left.\quad+u_{33}\left(\phi_{1}^{2}+\phi_{2}^{2}\right)\right]+q_{44}\left(u_{23} \phi_{2} \phi_{3}+u_{31} \phi_{1} \phi_{3}+u_{12} \phi_{2} \phi_{1}\right)\right\} .
\end{gathered}
$$

Here $\phi_{\alpha}$ and $u_{\alpha \beta}$ are the Cartesian components of the fluctuating polarization and strain, $\phi_{\alpha q}$ stands for the Fourier transform of $\phi_{\alpha}(x), n_{\alpha}=\frac{q_{\alpha}}{q}, C$ is the Curie constant, $\Delta^{2} \sim \epsilon_{0}^{-1} \sim s a^{-1}$ is the dipole gap in the fluctuation spectrum, $a$ being a lattice constant. Contrary to the original Landau-Devonshire expansion, the Hamiltonian (1-3) accounts for the inhomogeneous fluctuations of the polarization and elastic strains making it possible to explore the fluctuation effects in $\mathrm{BaTiO}_{3}$.

Dealing with the first-order phase transition, we are in a position to study the fluctuation effects in the region where thermal fluctuations of the order parameter are weak. Hence, in what follows we limit ourselves by the calculation of the first-order fluctuation corrections to the quantities of interest. The quantities to be found are the full four-leg and six-leg vertices, reducing, under zero external momenta, to the effective ("dressed") quartic $B_{i}$ and sextic $\Gamma_{i}$ coefficients, entering the Landau-Devonshire expansion. Among five Feynman diagrams representing the lowest-order non-trivial terms in the perturbative expansions of the vertices 
mentioned, only two one-loop graphs give contributions that rapidly, as $\left(T-T_{0}\right)^{-1 / 2}$, grow up approaching the transition point. These graphs have an obvious structure and may be calculated in a standart way using the propagator

$$
G_{\alpha \beta}(q)=\frac{k_{B} T\left(\delta_{\alpha \beta}-n_{\alpha} n_{\beta}\right)}{\left(C \epsilon_{0}\right)^{-1}\left(T-T_{0}\right)+s^{2} q^{2}},
$$

with a longitudinal part fully neglected. The results for the quartic couplings $B_{1}$ and $B_{2}$ are found to be:

$$
\begin{aligned}
& B_{1}=\beta_{1}-\frac{k_{B} T \sqrt{C \epsilon_{0}}}{10 \pi s^{3} \sqrt{T-T_{0}}}\left(24 \beta_{1}^{2}+4 \beta_{1} \beta_{2}+6 \beta_{2}^{2}\right), \\
& B_{2}=\beta_{2}-\frac{k_{B} T \sqrt{C \epsilon_{0}}}{10 \pi s^{3} \sqrt{T-T_{0}}}\left(3 \beta_{1}^{2}+18 \beta_{1} \beta_{2}+13 \beta_{2}^{2}\right),
\end{aligned}
$$

The polynomials in brackets are easily seen to coincide with those of the one-loop contributions to the renormalization-group $\beta$-functions of the cubic ferroelectric [8.9]. It is not surprising since, in fact, the same integrals and tensor convolutions are evaluated in both cases. The fluctuation correction to $B_{1}$ given by the first equation (5) is consistent with the results of Vaks [5,10], who first evaluated this correction and showed that it is essential for the case of $\mathrm{BaTiO}_{3}$.

To proceed further, we have to estimate the "bare" coupling constants $\beta_{1}$ and $\beta_{2}$ for $\mathrm{BaTiO}_{3}$ trusting upon the experimental data available. Aiming to extract the necessary information from experiments, one should realize that i) what is measured are not bare but dressed couplings with the fluctuation contributions included, and ii) in experiments, the Landau-Devonshire coefficients are measured for stress-free (not clamped) crystals. Hence, in order to estimate $\beta_{1}$ and $\beta_{2}$, we have first to express them via their analogs for free crystal, $\beta_{1}^{f}$ and $\beta_{2}^{f}$. This problem is solved by evaluation of the elastic strains caused by the non-zero uniform polarization and consequent renormalization of coefficients in the relevant LandauDevonshire expansion [1]. All the elastic and electrostrictive moduli are known for $\mathrm{BaTiO}_{3}$ [7], making corresponding calculations straightforward. The final result is as follows:

$$
\beta_{1}=\beta_{1}^{f}+7.4 \cdot 10^{8} V m^{5} C^{-3}, \quad \beta_{2}=\beta_{2}^{f}-2.3 \cdot 10^{8} V m^{5} C^{-3},
$$


Since in the vicinity of $T_{c}$ the elastic and electrostrictive moduli weakly depend on temperature, similar relations should be valid for the fluctuation modified (dressed) quartic coefficients $B_{1}, B_{2}, B_{1}^{f}$, and $B_{2}^{f}$.

At the transition point $\left(T_{c}=393 \mathrm{~K}\right), B_{1}^{f}=-2.0 \cdot 10^{8} \mathrm{Vm}^{5} \mathrm{C}^{-3}, B_{2}^{f}=1.6 \cdot 10^{8} \mathrm{Vm}^{5} \mathrm{C}^{-3}$ [6.7] and, hence, $B_{1}=5.4 \cdot 10^{8} \mathrm{Vm}^{5} \mathrm{C}^{-3}, B_{2}=-0.7 \cdot 10^{8} \mathrm{Vm}^{5} \mathrm{C}^{-3}$. It is easy to see that for $B_{1}>B_{2}$ the rhombohedral phase has lower free energy than the tetragonal one, provided the six-order form is isotropic, i. e. does not influence their competition. Hence, in the clamped crystal the quartic form of the Landau-Devonshire expansion strongly favours the phase transition into the rhombohedral phase. The same is true for higher temperatures $T=415 \mathrm{~K}$ and $T=423 \mathrm{~K}$, where $B_{1}^{f}=-1.3 \cdot 10^{8} \mathrm{Vm}^{5} \mathrm{C}^{-3}$ [2], $B_{1}=6.1 \cdot 10^{8} \mathrm{Vm}^{5} \mathrm{C}^{-3}$ and $B_{1}^{f}=-1.0 \cdot 10^{8} \mathrm{Vm}^{5} \mathrm{C}^{-3}$ 迥, $B_{1}=6.4 \cdot 10^{8} \mathrm{Vm}^{5} \mathrm{C}^{-3}$, respectively, with $B_{2}^{f}$ and $B_{2}$ kept unchanged.

Now we are ready to estimate to what extend the fluctuations can modify the behaviour of barium titanate in the vicinity of $T_{c}$. As is seen from Eqs.(55), the quartic Landau-Devonshire coefficients should vary with temperature in a similar way, provided the fluctuation corrections to them are of the same order of magnitude. Let us compare the magnitudes of the fluctuation terms $B_{2}^{(1)}$ and $B_{1}^{(1)}$ within the domain where parameters $\beta_{1}$ and $\beta_{2}$ have relevant values and signs. The quantity characterizing relative weights of $B_{2}^{(1)}$ and $B_{1}^{(1)}$ is their ratio, that can be found directly from Eqs. (5). The plot of the ratio $R^{(1)}=\frac{B_{2}^{(1)}}{B_{1}^{(1)}}$ as a function of $r=\frac{\beta_{1}}{\beta_{2}}$ is shown in Fig. 1. Analyzing this function, one can find, in particular, that $\left|\frac{B_{2}^{(1)}}{B_{1}^{(1)}}\right|$ does not exceed 0.1 if $\frac{\beta_{1}}{\beta_{2}}$ lies between -28 and -2.3 .

Let us estimate further $\frac{\beta_{1}}{\beta_{2}}$ in $\mathrm{BaTiO}_{3}$. Since, according to experiments, $B_{1}$ varies with the temperature appreciably, an estimate for $\beta_{1}$ is expected to have somewhat limited accuracy. To keep the perturbation theory more or less meaningful, one should adopt that the fluctuation term $B_{1}^{(1)}$ is, at least, two times smaller than the value of $B_{1}$ at $T=T_{c}$. At the transition temperature $B_{1}=5.4 \cdot 10^{8} \mathrm{Vm}^{5} \mathrm{C}^{-3}$ and this coefficient was shown to increase when the temperature grows up. Hence, the limitation $\beta_{1} \leq 10^{9} \mathrm{Vm}^{5} \mathrm{C}^{-3}$ looks 
quite reasonable. Another quartic coefficient, $B_{2}$, does not depend on $T$ and the estimate $\beta_{2}=B_{2}=-0.7 \cdot 10^{8} \mathrm{Vm}^{5} \mathrm{C}^{-3}$ may be considered as an accurate one. Since, in any case, $\beta_{1}>B_{1}(T=393 K)=5.4 \cdot 10^{8} \mathrm{Vm}^{5} \mathrm{C}^{-3}$, we see that $-14<\frac{\beta_{1}}{\beta_{2}}<-8$ in barium titanate. It implies that, according to Fig. $1, B_{2}^{(1)}<0.08 B_{1}^{(1)}$, and the fluctuation correction $B_{2}^{(1)}$ is inevitably very small. This means that $B_{2}$ practically does not depend on $T$ and explanes why in $\mathrm{BaTiO}_{3}$ the Landau-Devonshire coefficient $B_{2}^{f}$ is insensitive to the temperature.

Apart from the smallness of $B_{2}^{(1)}$, the theory naturally accounts for the experimentally observed sign of the fluctuation contribution to $B_{1}$. Indeed, as seen from Eqs. (5), a sign of $B_{1}^{(1)}$ is completely controlled by the first term proportional to $\beta_{1}^{2}$ : big number 24 provides a positiveness of the polynomial in brackets avoiding any possibility for the rest terms to compete with the first one under any values of $\beta_{1}$ and $\beta_{2}$. Hence, the theory definitely predicts that the fluctuation correction $B_{1}^{(1)}$ is negative and, therefore, approaching $T_{c}$ from above $B_{1}$ and $B_{1}^{f}$ should vary downward. This conclusion is in agreement with experiments [2:3].

As we have already seen, the experimental data available do not allow to fix the true, temperature independent value of the Landau-Devonshire coefficient $\beta_{1}$. However, there are obvious requirements that enable us to improve the crude estimate for $\beta_{1}$ presented above. The theory developed, based on the first-order perturbative calculations, is believed to account for the main features of the behaviour of $\mathrm{BaTiO}_{3}$. Hence, it should explain the variation of $B_{1}^{f}(T)$ by, at least, $10^{8} \mathrm{Vm}^{5} \mathrm{C}^{-3}$ [2] and remain valid, at worst, at the semiquantitative level. It is possible to meet both requirements only accepting that the true value of $\beta_{1}$ is appreciably bigger than $6.4 \cdot 10^{8} \mathrm{Vm}^{5} \mathrm{C}^{-3}$ and appreciably smaller than $1.1 \cdot 10^{9} \mathrm{Vm}^{5} \mathrm{C}^{-3}$, i. e. it lies somewhere between $7 \cdot 10^{8} \mathrm{Vm}^{5} \mathrm{C}^{-3}$ and $10^{9} \mathrm{Vm}^{5} \mathrm{C}^{-3}$. As a result, the stress-free crystal should possess the Landau-Devonshire coefficient that obeys the inequalities $-0.4 \cdot 10^{8} \mathrm{Vm}^{5} \mathrm{C}^{-3}<\beta_{1}^{f}<2.6 \cdot 10^{8} \mathrm{Vm}^{5} \mathrm{C}^{-3}$.

The upper part of this interval is of particular interest. The point is that, whenever $\beta_{1}^{f}$ exceeds $1.6 \cdot 10^{8} \mathrm{Vm}^{5} \mathrm{C}^{-3}=\beta_{2}^{f}$, the crystal with the fluctuations being "switched off" would undergo a phase transition into the rhombohedral phase rather than into the tetragonal one. 
If this were true, i. e. the inequality $\beta_{1}^{f}>\beta_{2}^{f}$ took place, the tetragonal and orthorhombic phases in barium titanate would exist essentially due to the thermal fluctuations, while the rhombohedral phase would survive only at sufficiently low temperatures where the fluctuations are weak enough. Thus, the analysis based upon the effective Hamiltonian (1) turns out to support the conjecture about the fluctuation stabilization of the high-temperature ferroelectric phases in $\mathrm{BaTiO}_{3}$ formulated first within the microscopic theory [5].

This conjecture looks rather attractive. It is worthy to discuss it in more detail. It turns out that, apart from the results the of first-order calculations, there exist two extra arguments in favour of the scenario just described. The first is as follows. As we have already seen, the lowest-order correction to $\beta_{1}^{f}$ is considerable and, therefore, the higher-order fluctuation contributions can influence the results appreciably. The leading perturbative term shifting the first-order estimates is the second-order one and it has a sign opposite to that of $B_{1}^{(1)}$. One can show that account for the positive second-order fluctuation term in the course of fit of the experimental data [2] results in the shift of the estimated value of $\beta_{1}^{f}$ upward. Hence, within the refined theory the fluctuation origin of the tetragonal and orthorhombic phases in $\mathrm{BaTiO}_{3}$ would become more plausible.

The second argument deals with the fluctuation corrections to the six-order coefficients in the Landau-Devonshire expansion. Let us calculate them. Evaluating the six-leg one-loop diagram, we arrive to the following expressions for $\Gamma_{i}$ :

$$
\begin{aligned}
& \Gamma_{1}=\gamma_{1}-f(T)\left(120 \beta_{1} \gamma_{1}+6 \beta_{1} \gamma_{2}+10 \beta_{2} \gamma_{1}+18 \beta_{2} \gamma_{2}\right), \\
& \Gamma_{2}=\gamma_{2}-f(T)\left(5 \beta_{1} \gamma_{1}+63 \beta_{1} \gamma_{2}+2 \beta_{1} \gamma_{3}+15 \beta_{2} \gamma_{1}+63 \beta_{2} \gamma_{2}+6 \beta_{2} \gamma_{3}\right), \\
& \Gamma_{3}=\gamma_{3}-f(T)\left(18 \beta_{1} \gamma_{2}+24 \beta_{1} \gamma_{3}+54 \beta_{2} \gamma_{2}+58 \beta_{2} \gamma_{3}\right),
\end{aligned}
$$

where $f(T)=k_{B} T \sqrt{C \epsilon_{0}}\left(10 \pi s^{3} \sqrt{T-T_{0}}\right)^{-1}$.

Above formulas are written down for the physical case when the polarization vector has three Cartesian components. In fact, the fluctuation terms were calculated for the generic model with the $\mathrm{n}$-vector order parameter. It was done in order to reserve an opportunity for independent check of the results obtained. Indeed, as is well known, the model with 
cubic anisotropy possesses the special symmetry property under $n=2$ : if one turns the field $\left(\phi_{1}, \phi_{2}\right)$ by $45^{\circ}$ in its two-dimensional space, the coupling constants are transformed, but the structure of the Hamiltonian remains unchanged. This implies some exact symmetry relations between coupling constants [11], that interrelate dressed $\left(B_{i}, \Gamma_{i}\right)$ and bare $\left(\beta_{i}, \gamma_{i}\right)$ couplings and can be used to approve (or disregard) the results of perturbative calculations in the arbitrary order. Having made such a check up, we found that the n-vector analog of Eqs.(17) do obey these symmetry relations when $n=2$.

To estimate the magnitudes of fluctuation corrections $\Gamma_{i}^{(1)}$, the experimental information about $\gamma_{i}$ is necessary. The experiments, however, were carried out in the ordered (tetragonal) phase and yielded, in particular, strong dependence of $\Gamma_{1}$ on temperature. This temperature dependence was already mentioned to be so dramatic that, being extrapolated to the paraelectric region, makes the Landau-Devonshire form unstable for $T>T_{c}+50 K$. It is hardly believed therefore that the experimental data available can be used to extract more or less reliable estimates for $\gamma_{i}$.

In such a situation it is natural to analyze the general structure of the correction terms $\Gamma_{i}^{(1)}$ in Eqs. (7), aiming to find some conclusions that are insensitive to the concrete values of $\gamma_{i}$. Let us proceed, accepting that $\gamma_{1} \sim \gamma_{2} \sim \gamma_{3}$. As we have already found, in barium titanate $\left|\beta_{2}\right|<<\left|\beta_{1}\right|$. It means that the magnitudes of $\Gamma_{i}^{(1)}$ are determined, in fact, by the terms in Eqs. (7) containing $\beta_{1}$. The numerical coefficients before these terms are seen to be markedly different. The biggest one (120) stands in the expression for $\Gamma_{1}^{(1)}$, making $\Gamma_{1}$ stronger dependent on temperature than two other coefficients. Moreover, the structure of $\Gamma_{1}^{(1)}$ fixes its sign. Since $\beta_{1}>0, \Gamma_{1}^{(1)}$ is negative and the fluctuations diminish the Landau-Devonshire coefficient $\Gamma_{1}$ provided $\gamma_{1}>0$. The positiveness of $\gamma_{1}$ is, in its turn, inevitable, because this coefficient is responsible for the global stability of the system outside the fluctuation region.

So, we see that approaching $T_{c}$ the coefficient $\Gamma_{1}$ decreases more rapidly than $\Gamma_{2}$ and $\Gamma_{3}$. On the other hand, as one can see, the smaller $\Gamma_{1}$, the more stable is the tetragonal phase. Hence, the fluctuations modify the six-order form in the Landau-Devonshire expansion in a 
way that favours the transition into the tetragonal phase. It confirms the conjecture about the fluctuation stabilization of the phases lying between the cubic and rhombohedral ones at the phase diagram of barium titanate.

To conclude, we have shown that the temperature dependence of the quartic LandauDevonshire coefficients in a paraelectric phase of $\mathrm{BaTiO}_{3}$ can be explained as the fluctuation effect. The theory developed naturally accounts for a signs of the temperature variations of $B_{1}$ and makes it clear why the second coefficient, $B_{2}$, is temperature independent. The conjecture was formulated that the true Landau-Devonshire form for $\mathrm{BaTiO}_{3}$ with the temperature independent coefficients favours the transition from cubic into the rhombohedral phase, and it is the thermal fluctuations what stabilizes the tetragonal and orthorhombic phases and provides the space for them at the phase diagram.

The authors acknowledge the financial support of the Russian Foundation for Basic Research under Grant No. 01-02-17048 (A.I.S.), the Ministry of Education of Russian Federation under Grant No. E00-3.2-132 (A.I.S.), and the Swiss National Science Foundation (A.K.T.). A.I.S. has benefitted from the hospitality of the Laboratoire de Céramique, Ecole Polytechnique Fédéral de Lausanne, where this research was done. 


\section{REFERENCES}

[1] A.F. Devonshire, Phil. Mag. 40, 1040 (1949)1; 42, 1065 (1951).

[2] M.E. Drougard, R. Landauer, and D.R. Young, Phys. Rev. 98, 1010 (1955).

[3] E.J. Huibregtse and D.R. Young, Phys. Rev. 103, 1705 (1956).

[4] W.R. Buessem, L.E. Cross, and A.K. Goswami, J. Am. Ceram. Soc. 49, 33 (1966).

[5] V.G. Vaks, Introduction to the Microscopic Theory of Ferroelectrics [in Russian] (Nauka, Moscow, 1973).

[6] A.J. Bell and L.E. Cross, Ferroelectrics 59, 197 (1984).

[7] A.J. Bell, J. Appl. Phys. 89, 3907 (2001).

[8] A.I. Sokolov, Pis'ma v Zh.E.T.F. 22, 199, (1975) [JETP Lett. 22, 92 (1975)].

[9] A.I. Sokolov, A.K. Tagantsev, Zh.E.T.F. 76, 181 (1979) [Sov. Phys. JETP 49, 92 (1979)]

[10] V.G. Vaks, Zh.E.T.F. 58, 296 (1970).

[11] D.V. Pakhnin and A.I. Sokolov, Phys. Rev. B 64094407 (2001).

\section{CAPTION}

Fig. 1. The ratio of the first-order fluctuation corrections $R^{(1)}=\frac{B_{2}^{(1)}}{B_{1}^{(1)}}$ as a function of the ratio of bare coupling constants $r=\frac{\beta_{1}}{\beta_{2}}$. 